\title{
LIMIT POINTS OF SUBSEQUENCES
}

\section{R. CREIGHTON BUCK ${ }^{1}$}

1. Introduction. In a previous paper [2], ${ }^{2}$ it was shown that for simple sequences of real numbers, divergence of a sequence implies divergence of almost every subsequence. The proof given there required in an essential way that the space be metric. The purpose of this note is to show that the above result holds for multiple sequences in an $L^{*}$ space. If the space is compact separable metric, even more is true: the set of limit points of almost every subsequence coincides with the set of limit points of the original sequence.

2. Notation. We denote by $x=x[n]=x\left[n_{1}, n_{2}, \cdots, n_{r}\right]$, where $n_{k}=1,2, \cdots$, an arbitrary $r$-tuple sequence with terms in a space $M$. Likewise, $x^{\prime}=x_{\lambda}[n]=x\left[\lambda_{1}\left(n_{1}\right), \lambda_{2}\left(n_{2}\right), \cdots, \lambda_{r}\left(n_{r}\right)\right]$ will denote an arbitary subsequence of $x$; here, for any fixed $k, \lambda_{k}(1), \lambda_{k}(2), \ldots$ form an increasing sequence of integers. This is the natural generalization of subsequences $x_{\lambda_{n}}$ of the simple sequence $x_{n}$.

Let $S$ be the set of all sequences $s=\left(s_{\alpha}\right)$ composed of 0 's and 1's, containing infinitely many 1 's. This represents in the usual manner the class of subsequences of a simple sequence $[1, p .788]$ that is $s_{n}=1$ if $x_{n}$ is chosen, and 0 if $x_{n}$ is omitted. A product measure can be defined in $\delta\left[4\right.$, p. $420 ; 5$, p. 144]. ${ }^{3}$

Then

$$
\Im=\delta \times \delta \times \cdots \times S
$$

is the class of all subsequences of an $r$-tuple sequence. Measure is defined in $\mathfrak{S}$ as the product measure over $\mathcal{S}$.

We assume that some definition of convergence is given for sequences in $M$. Limit points are then defined as

$$
P x=\hat{p}\left[\lim x^{\prime}=p \text { for some subsequence } x^{\prime} \text { of } x\right] \text {. }
$$

We recall the defining conditions of the Fréchet limit spaces $[3, p$. 77]:

(L) $\lim x=p$ implies $\lim x^{\prime}=p$ for every subsequence $x^{\prime}$ of $x$.

$\left(L^{*}\right) \lim x=p$ if and only if $p \in P x^{\prime}$ for every subsequence $x^{\prime}$ of $x$.

$L^{*}$ is the "star-convergence" of Urysohn.

Presented to the Society, October 30, 1943, under the title Multiple sequences; received by the editors December 3, 1943.

1 Society of Fellows, Harvard University.

2 Numbers in brackets refer to the references listed at the end of the paper.

${ }^{3} S \subseteq S^{*}=S \times S \times S \times S \times \cdots$, where $S=\{0,1\}$. Both $\mathcal{S}$ and $S^{*}$ have measure 1. $S$ can be mapped, with measure preserved, on the real numbers $0<t \leqq 1$. 
3. Limit points. The following lemma is essential.

LEMma. Almost every $s \in \mathcal{S}$ coincides with any fixed $t \in \mathcal{S}$ in an infinite number of 1 coordinates.

Set $s=\left(s_{\alpha}\right)$ and $t=\left(t_{\alpha}\right) ; t_{\alpha}$ is 1 for an infinite set of $\alpha$ 's. Let

$$
\begin{aligned}
& F_{n}=s\left[s_{\alpha} t_{\alpha}=0 \text { for all } \alpha>n\right], \\
& C_{n}=s\left[\sum s_{\alpha} t_{\alpha}=n\right] .
\end{aligned}
$$

Then $F_{n}$ is an elementary subset of $S$, and furthermore $m\left(F_{n}\right)=0$; since $C_{n} \subseteq \mathrm{U}_{k \geqq n} F_{k}, m\left(C_{n}\right)=0$. If $C=\bigcup C_{n}$, then $m(C)=0$ so that $m\left(C^{\prime}\right)$ $=1$ where

$$
C^{\prime}=\hat{s}\left[\sum s_{\alpha} t_{\alpha}=\infty\right] .
$$

Theorem 1. If $M$ is an $L$ space and $p \in P x$, then $p \in P x^{\prime}$ for almost every subsequence $x^{\prime}$ of $x$.

If $p \in P x$, then for some subsequence $x^{\prime \prime}$ of $x, \lim x^{\prime \prime}=p$. Let $\tau$ be the point of $\subseteq$ corresponding to $x^{\prime \prime}$, and $\tau=\left(t^{1}, t^{2}, t^{3}, \cdots, t^{r}\right)$. By the lemma, for each $k$ there is a set $\Sigma_{k}$ having measure 1 , composed of points $s^{k}=\left(s_{\alpha}^{k}\right)$ of $S$ whose coordinates coincide with those of $t^{k}=\left(t_{\alpha}^{k}\right)$ at an infinite number of 1 's. Then

$$
\Sigma=\Sigma_{1} \times \Sigma_{2} \times \cdots \times \Sigma_{r}
$$

is a subset of $\subseteq$ composed of points $\sigma=\left(s^{1}, s^{2}, s^{3}, \cdots, s^{r}\right)$ each of which coincides with $\tau$ in an infinite number of components. $\Sigma$ then corresponds to a set of subsequences $x^{\prime}$ of $x$ each of which has a subsequence in common with $x^{\prime \prime}$. By $(L)$, this common subsequence converges to $p$, and $p \in P x^{\prime}$. This is true for almost every subsequence $x^{\prime}$, for $m(\Sigma)=1$.

THEOREM 2. If $M$ is an $L^{*}$ space with unique limits, then if $x$ is divergent, so is almost every subsequence $x^{\prime}$ of $x .^{4}$

Either $P x$ is void, or $P x=\{p\}$, or $P x$ contains at least two distinct points. If $P x$ is void, then every subsequence of $x$ is divergent. If $P x$ contains distinct points $p$ and $q$, then by the previous theorem, these are limit points of almost every subsequence, and since limits are unique, these are in turn divergent. Finally, if $x$ is divergent and $P x=\{p\}$, there must be a subsequence $x^{\prime \prime}$ of $x$ for which $P x^{\prime \prime}$ is void. As in the proof of Theorem 1, almost every subsequence $x^{\prime}$ of $x$ has a subsequence in common with $x^{\prime \prime}$, and must therefore be divergent.

\footnotetext{
${ }^{4}$ The statements of this and the following theorem were altered at the suggestion of Dr. M. M. Day.
} 
We now suppose that closure of subsets of $M$ is defined.

Theorem 3. If $M$ is an $L$ space and $P x$ is separable, then $\bar{P} x^{\prime}=\bar{P} x$ for almost every subsequence $x^{\prime}$ of $x$.

Choose a countable dense set $E \subset P x$. Let $A_{p}$ be the class of sub-

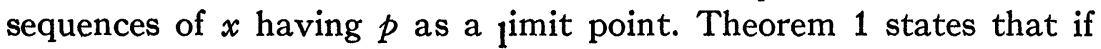
$p \in P x, m\left(A_{p}\right)=1$. Set $A=\bigcap_{p \in E} A_{p} ;$ since $E$ is countable, $m(A)=1$. Thus, for almost every subsequence $x^{\prime}$ of $x, E \subset P x^{\prime} \subset P x$. Closing these sets, we have the theorem.

If $M$ is a neighborhood space, and we define convergence in the usual way, $M$ becomes an $L^{*}$ space. Thus, Theorem 1 holds for multiple sequences in any topological space. If, further, $M$ obeys the Hausdorff separation axiom, limits are unique and Theorem 2 holds. If $M$ is a topological space with second countability, then $P x$ is separable, and Theorem 3 holds.

CoRollary 1. If $M$ is a topological space with second countability, such that the interior of any neighborhood of a point contains a neighborhood of that point, then for simple sequences $x, P x=P x^{\prime}$ for almost all subsequences $x^{\prime}$ of $x$.

The additional assumption implies that for simple sequences, the set of limit points is closed. (It might be noted that, as subsequences and limit points are here defined, the set of limit points of a multiple sequence need not be closed!)

CoRollary 2. If, in addition to the assumptions above, $M$ is also a group, then almost every bracketing of the series $\sum a_{n}$ leaves the set of limit points unchanged.

As in [2], bracketing a series is equivalent to selecting a subsequence.

\section{REFERENCES}

1. Birnbaum and Zuckerman, On the properties of a collective, Amer. J. Math. vol. 62 (1940) pp. 787-791.

2. R. C. Buck and Harry Pollard, Convergence and summability properties of subsequences, Bull. Amer. Math. Soc. vol. 49 (1943) pp. 924-931.

3. Kuratowski, Topologie. I, Warsaw, 1933.

4. van Kampen, Infinite product measures and infinite convolutions, Amer. J. Math. vol. 62 (1940) pp. 417-448.

5. C. Visser, Law of nought or one, Studia Math. vol. 7 (1938) pp. 143-159.

HARVARD UNIVERSITY 